Benlian Lv*

\title{
Crystal structure of bis $\left(\mu_{2}-4\right.$-iodopyridine-2,6- dicarboxylato- $\left.\mathrm{K}^{3} \mathrm{O}: \mathrm{N}: \mathrm{O}^{\prime}\right)$-bis(4-iodopyridine-2,6- dicarboxylato-k $\left.\mathrm{K}^{3} \mathrm{O}: \mathrm{N}: \mathrm{O}^{\prime}\right)$-bis $\left(\mu_{2}-1-(4-\mathrm{pyridyl})\right.$ piperazine- $\kappa^{2} N: N^{\prime}$ )-hexa-aqua-tetra-copper(II), $\mathrm{C}_{46} \mathrm{H}_{46} \mathrm{Cu}_{4} \mathrm{I}_{4} \mathrm{~N}_{10} \mathrm{O}_{22}$
}

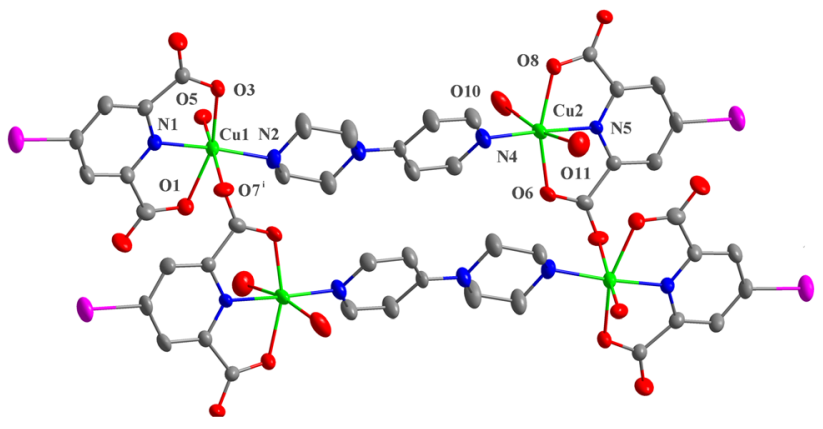

https://doi.org/10.1515/ncrs-2021-0166

Received May 3, 2021; accepted May 31, 2021; published online June 24, 2021

\begin{abstract}
$\mathrm{C}_{46} \mathrm{H}_{46} \mathrm{Cu}_{4} \mathrm{I}_{4} \mathrm{~N}_{10} \mathrm{O}_{22}$, monoclinic, $P 2_{1} / c$ (no. 14), $a=10.6637(2) \AA, b=18.8249(6) \AA, c=14.8626(5) \AA$, $\beta=93.853(3)^{\circ}, Z=2, V=2976.81(15) \AA^{3}, R_{g t}(F)=0.0361$, $w R_{\text {ref }}\left(F^{2}\right)=0.0726, T=291 \mathrm{~K}$.
\end{abstract}

CCDC no.: 2081282

The molecular structure is shown in the figure. Table 1 contains crystallographic data and Table 2 contains the list of the atoms including atomic coordinates and displacement parameters.

\section{Source of material}

The title compound was synthesized via the reaction of 4-iodopyridine-2,6-dicarboxylic acid (ipydc) (29.2 mg, $0.1 \mathrm{mmol})$, 1-(4-pyridyl)piperazine (pypi) (16.3 mg,

*Corresponding author: Benlian Lv, School of Chemical Engineering and Pharmaceutics, Henan University of Science and Technology, Luoyang 471023, China, E-mail: bl9901343@163.com. https://orcid. org/0000-0002-7609-1215
Table 1: Data collection and handling.

\begin{tabular}{|c|c|}
\hline Crystal: & Green block \\
\hline Size: & $0.32 \times 0.30 \times 0.28 \mathrm{~mm}$ \\
\hline Wavelength: & Mo $K \alpha$ radiation $(0.71073 \AA$ ) \\
\hline$\mu:$ & $3.57 \mathrm{~mm}^{-1}$ \\
\hline Diffractometer, scan mode: & SuperNova, $\omega$ \\
\hline$\theta_{\max }$, completeness: & $25.0^{\circ},>99 \%$ \\
\hline$N(h k l)_{\text {measured }}, N(h k l)_{\text {unique }}, R_{\text {int }}:$ & $17,298,5227,0.032$ \\
\hline Criterion for $I_{\mathrm{obs}}, N\left(h k l_{\mathrm{gt}}\right.$ : & $I_{\text {obs }}>2 \sigma\left(I_{\text {obs }}\right), 4340$ \\
\hline$N(\text { param })_{\text {refined }}:$ & 393 \\
\hline Programs: & $\begin{array}{l}\text { CrysAlis }{ }^{\text {PRO }}[1] \text {, SHELX [2, 3], } \\
\text { Olex2 [4] }\end{array}$ \\
\hline
\end{tabular}

$0.1 \mathrm{mmol}), \mathrm{Cu}(\mathrm{OAc})_{2} \cdots \mathrm{H}_{2} \mathrm{O}(20.0 \mathrm{mg}, 0.1 \mathrm{mmol})$ in $4 \mathrm{~mL}$ deionized water. The mixture was placed in a $23 \mathrm{ml}$ Teflon lined stainless steel reactor and then heated at $343 \mathrm{~K}$ for two days to afford green block crystals in a yield of $81 \%$.

\section{Experimental details}

Hydrogen atoms were placed in their geometrically idealized positions and constrained to ride on their parent atoms.

\section{Comment}

The design and synthesis of inorganic-organic coordination compounds have been an active area because such compounds could exhibit a variety of unique functional applications [5-8]. At present, the appropriate choice of organic ligands and central metal ions is one of the most effective ways to construct these compounds $[9,10]$. In this regard, versatile pyridine polycarboxylic acids have been widely used as excellent candidates owing to their high structural stability and versatile coordination modes. As a member of the pyridine polycarboxylate 
Table 2: Fractional atomic coordinates and isotropic or equivalent isotropic displacement parameters $\left(\AA^{2}\right)$.

\begin{tabular}{|c|c|c|c|c|}
\hline Atom & $x$ & $y$ & $z$ & $U_{\text {iso }}{ }^{*} / U_{\text {eq }}$ \\
\hline 11 & $1.60627(3)$ & $0.38574(2)$ & $0.92771(2)$ & $0.04945(12)$ \\
\hline 12 & $-0.43199(3)$ & $0.81703(2)$ & $0.33537(3)$ & $0.04988(12)$ \\
\hline Cu1 & $1.02672(5)$ & $0.48060(3)$ & $0.76534(4)$ & 0.02909 (14) \\
\hline Cu2 & $0.13075(5)$ & $0.68971(3)$ & $0.48657(4)$ & $0.03172(15)$ \\
\hline 01 & $1.0033(3)$ & $0.37132(16)$ & $0.7825(2)$ & $0.0328(7)$ \\
\hline 02 & $1.1106(3)$ & $0.27713(17)$ & $0.8399(3)$ & 0.0479 (9) \\
\hline 03 & $1.1155(3)$ & $0.57880(16)$ & $0.7648(2)$ & $0.0327(7)$ \\
\hline 04 & $1.2975(3)$ & $0.63106(17)$ & $0.8096(2)$ & 0.0457 (9) \\
\hline 05 & $0.9234(3)$ & $0.50655(17)$ & $0.8874(2)$ & $0.0386(8)$ \\
\hline H5A & 0.9650 & 0.5339 & 0.9239 & $0.058^{*}$ \\
\hline H5B & 0.9143 & 0.4700 & 0.9198 & $0.058^{*}$ \\
\hline 06 & $0.0417(3)$ & 0.59759 (15) & $0.4487(2)$ & $0.0341(8)$ \\
\hline 07 & $-0.1372(3)$ & $0.55836(16)$ & $0.3778(2)$ & $0.0375(8)$ \\
\hline 08 & $0.1642(3)$ & $0.79722(15)$ & $0.5026(2)$ & $0.0331(7)$ \\
\hline 09 & $0.0676(3)$ & $0.90154(16)$ & $0.4864(2)$ & $0.0437(9)$ \\
\hline 010 & $0.0916(3)$ & 0.69317 (19) & $0.6399(2)$ & 0.0509 (10) \\
\hline $\mathrm{H} 10 \mathrm{~A}$ & 0.0837 & 0.6516 & 0.6612 & $0.076^{\star}$ \\
\hline H10B & 0.0205 & 0.7118 & 0.6471 & $0.076^{*}$ \\
\hline 011 & $0.2037(4)$ & $0.7115(2)$ & $0.3271(3)$ & $0.0626(11)$ \\
\hline $\mathrm{H} 11 \mathrm{~A}$ & 0.2230 & 0.7533 & 0.3114 & $0.094^{\star}$ \\
\hline H11B & 0.1467 & 0.6952 & 0.2899 & $0.094^{*}$ \\
\hline N1 & $1.1879(3)$ & $0.45622(19)$ & $0.8222(2)$ & $0.0276(8)$ \\
\hline N2 & $0.8732(3)$ & $0.5010(2)$ & $0.6888(2)$ & $0.0324(9)$ \\
\hline $\mathrm{H} 2$ & 0.8840 & 0.4729 & 0.6343 & 0.039 * \\
\hline N3 & $0.6344(3)$ & $0.5600(2)$ & $0.6248(3)$ & $0.0340(9)$ \\
\hline N4 & $0.2911(3)$ & $0.6482(2)$ & $0.5263(3)$ & $0.0346(9)$ \\
\hline N5 & $-0.0283(3)$ & $0.72679(18)$ & $0.4421(2)$ & $0.0245(8)$ \\
\hline C1 & 1.0985 (4) & $0.3405(3)$ & $0.8206(3)$ & $0.0324(11)$ \\
\hline C2 & $1.2113(4)$ & $0.3889(2)$ & $0.8416(3)$ & $0.0288(10)$ \\
\hline C3 & $1.3303(4)$ & $0.3674(3)$ & $0.8729(3)$ & 0.0349 (11) \\
\hline H3 & 1.3471 & 0.3200 & 0.8864 & $0.042^{\star}$ \\
\hline $\mathrm{C} 4$ & $1.4242(4)$ & $0.4187(3)$ & $0.8837(3)$ & $0.0333(11)$ \\
\hline C5 & $1.3975(4)$ & $0.4889(2)$ & $0.8635(3)$ & 0.0329 (11) \\
\hline H5 & 1.4596 & 0.5235 & 0.8707 & $0.039^{\star}$ \\
\hline C6 & $1.2761(4)$ & $0.5068(2)$ & $0.8321(3)$ & $0.0278(10)$ \\
\hline C7 & $1.2284(4)$ & $0.5789(2)$ & $0.8011(3)$ & $0.0299(10)$ \\
\hline $\mathrm{C} 8$ & $0.8619(4)$ & $0.5718(3)$ & $0.6560(4)$ & 0.0474 (14) \\
\hline H8A & 0.9360 & 0.5825 & 0.6240 & $0.057^{*}$ \\
\hline H8B & 0.8625 & 0.6034 & 0.7075 & $0.057^{*}$ \\
\hline C9 & $0.7516(4)$ & $0.5883(3)$ & $0.5965(4)$ & 0.0507 (15) \\
\hline H9A & 0.7440 & 0.6395 & 0.5917 & $0.061^{\star}$ \\
\hline H9B & 0.7649 & 0.5701 & 0.5368 & $0.061^{*}$ \\
\hline C10 & $0.6429(4)$ & $0.4894(3)$ & $0.6615(4)$ & $0.0436(13)$ \\
\hline $\mathrm{H} 10 \mathrm{C}$ & 0.6391 & 0.4558 & 0.6120 & $0.052^{\star}$ \\
\hline H10D & 0.5703 & 0.4811 & 0.6960 & $0.052^{*}$ \\
\hline C11 & $0.7569(4)$ & $0.4752(3)$ & $0.7197(3)$ & $0.0399(12)$ \\
\hline H11C & 0.7459 & 0.4960 & 0.7784 & $0.048^{\star}$ \\
\hline $\mathrm{H} 11 \mathrm{D}$ & 0.7642 & 0.4243 & 0.7280 & $0.048^{\star}$ \\
\hline C12 & $0.5229(4)$ & $0.5868(2)$ & $0.5885(3)$ & $0.0253(10)$ \\
\hline C13 & $0.4070(4)$ & $0.5542(3)$ & $0.6027(3)$ & $0.0362(12)$ \\
\hline H13 & 0.4051 & 0.5122 & 0.6356 & $0.043^{*}$ \\
\hline C14 & $0.2961(4)$ & $0.5845(2)$ & $0.5678(3)$ & 0.0331 (11) \\
\hline H14 & 0.2215 & 0.5598 & 0.5731 & $0.040^{*}$ \\
\hline C15 & $0.4011(4)$ & $0.6790(3)$ & $0.5087(4)$ & $0.0420(13)$ \\
\hline H15 & 0.3999 & 0.7194 & 0.4726 & $0.050 *$ \\
\hline
\end{tabular}

Table 2: (continued)

\begin{tabular}{lrrrr}
\hline Atom & $\boldsymbol{x}$ & $\boldsymbol{y}$ & $\boldsymbol{z}$ & $\boldsymbol{U}_{\text {iso* }} \boldsymbol{U}_{\text {eq }}$ \\
\hline C16 & $0.5166(4)$ & $0.6521(3)$ & $0.5429(3)$ & $0.0430(13)$ \\
H16 & 0.5899 & 0.6776 & 0.5355 & $0.052^{*}$ \\
C17 & $-0.0694(4)$ & $0.6049(2)$ & $0.4119(3)$ & $0.0280(10)$ \\
C18 & $-0.1161(4)$ & $0.6813(2)$ & $0.4100(3)$ & $0.0239(9)$ \\
C19 & $-0.2322(4)$ & $0.7057(2)$ & $0.3777(3)$ & $0.0297(10)$ \\
H19 & -0.2943 & 0.6744 & 0.3555 & $0.036^{*}$ \\
C20 & $-0.2539(4)$ & $0.7782(2)$ & $0.3791(3)$ & $0.0304(11)$ \\
C21 & $-0.1606(4)$ & $0.8250(2)$ & $0.4108(3)$ & $0.0321(11)$ \\
H21 & -0.1746 & 0.8737 & 0.4111 & $0.039^{*}$ \\
C22 & $-0.0455(4)$ & $0.7966(2)$ & $0.4420(3)$ & $0.0264(10)$ \\
C23 & $0.0713(4)$ & $0.8358(2)$ & $0.4800(3)$ & $0.0292(10)$ \\
\hline
\end{tabular}

ligands, ipydc contains several coordination sites, which can generate unusual structures via its multiple bonding interactions $[11,12]$.

The crystallographic independent unit contains two $\mathrm{Cu}$ (II) ions and the coordination polyhedron for each metal is best described as a slightly distorted octahedron. The basal plane is occupied by the chelating tridentate ipydc donors, and by the pypi nitrogen atoms. The $\mathrm{Cu}(1)$ atom has a carboxylate oxygen atom and a water molecule at the apex of the octahedron, respectively. On the other hand, the $\mathrm{Cu}(2)$ atom has two water molecules at the apex of the octahedron. In the complex, ipydc ligand with a chelating/bridging tridentate/monodentate coordination mode and the pypi ligand link $\mathrm{Cu}^{2+}$ centers to generate a tetranuclear structure (see the figure). Moreover, there are hydrogen bonding interactions between coordinated water molecules and carboxyl oxygen atoms, between nitrogen atom of piperazine and carboxyl oxygen atoms. In addition, the structure also contains $0 \cdots \mathrm{I}$ halogen bond interactions between carboxyl oxygen atoms and iodine atoms. All non-covalent interactions mentioned above contribute to the formation of the supramolecular architecture.

Author contributions: The author has accepted responsibility for the entire content of this submitted manuscript and approved submission.

Research funding: $\mathrm{PhD}$ Startup Fund of HAUST (13480061).

Conflict of interest statement: The author declares no conflicts of interest regarding this article.

\section{References}

1. Agilent Technologies. CrysAlis ${ }^{\text {PRo }}$ Software System (version 1.171.40.53); Agilent Technologies UK Ltd: Oxford, UK, 2019. 
2. Sheldrick G. M. SHELXTL - integrated space-group and crystalstructure determination. Acta Crystallogr. 2015, A71, 3-8.

3. Sheldrick G. M. Crystal structure refinement with SHELXL. Acta Crystallogr. 2015, C71, 3-8.

4. Dolomanov O. V., Bourhis L. J., Gildea R. J., Howard J. A. K., Puschmann H. OLEX2: a complete structure solution, refinement and analysis program. J. Appl. Crystallogr. 2009, 42, 339-341.

5. Manna S., Ribas J., Zangrando E., Chaudhuri N. Supramolecular networks of dinuclear copper(II): synthesis, crystal structure and magnetic study. Inorg. Chim. Acta 2007, 360, 2589-2597.

6. Du X.-G., Mi G., Liu J.-C., Zhang J. A two-dimensional Zn(II) coordination polymer with a three-dimensional supramolecular architecture comprising 5-dimethylamino-isophthalic acid and 1,3-bis(4- pyridyl)propane. Mol. Cryst. Liq. Cryst. 2016, 624, 44-50.

7. Li Z.-H., Xue L.-P., Mu Y.-J., Zhao B.-T. Viologen-derived material showing photochromic, visually oxygen responsive, and photomodulated luminescence behaviors. CrystEngComm 2021, 23, 1019-1024.
8. Jiang X., Li Z., Zhai Y., Yan G., Xia H., Li Z. Porous coordination polymers based on azamacrocyclic complex: syntheses, solvent induced reversible crystal-to-crystal transformation and gas sorption properties. CrystEngComm 2014, 16, 805-813.

9. Manna S., Ribas J., Zangrando E., Chaudhuri N. Dipyridyl bridged coordination polymers of $\mathrm{Cu}(\mathrm{II})$ : synthesis, crystal structure and magnetic study. Polyhedron 2007, 26, 4923-4928.

10. Du X., Wang C. Crystal structure of bis(6-aminopyridine2-carboxylato- $\mathrm{K}^{2} \mathrm{O}, N$ )-copper(II), $\mathrm{C}_{12} \mathrm{H}_{10} \mathrm{O}_{6} \mathrm{~N}_{4} \mathrm{Cu}$. Z. Kristallogr. $N$. Cryst. Struct. 2020, 235, 1007-1008.

11. Felloni M., Blake A. J., Hubberstey P., Wilson C., Schröder M. Solvent control of supramolecular architectures derived from 4,4'-bipyridyl-bridged copper(II) dipicolinate complexes. Cryst. Growth Des. 2009, 9, 4685-4699.

12. Abdolmaleki S., Ghadermazi M., Fattahi A., Sheshmani S. Synthesis, characterization, spectral studies and cytotoxic effects of mixed-ligand mono and binuclear copper(II) complexes and their amide ligands. Inorg. Chim. Acta 2016, 443, 284-298. 